\title{
Transversal designs and induced decompositions of graphs *
}

\author{
Csilla Bujtás $^{1} \quad$ Zsolt Tuza ${ }^{1,2}$ \\ ${ }^{1}$ Department of Computer Science and Systems Technology \\ University of Pannonia \\ Veszprém, Hungary \\ 2 Alfréd Rényi Institute of Mathematics \\ Hungarian Academy of Sciences \\ Budapest, Hungary
}

Latest update on 2015-01-14

\begin{abstract}
We prove that for every complete multipartite graph $F$ there exist very dense graphs $G_{n}$ on $n$ vertices, namely with as many as $\left(\begin{array}{l}n \\ 2\end{array}\right)-c n$ edges for all $n$, for some constant $c=c(F)$, such that $G_{n}$ can be decomposed into edge-disjoint induced subgraphs isomorphic to $F$. This result identifies and structurally explains a gap between the growth rates $O(n)$ and $\Omega\left(n^{3 / 2}\right)$ on the minimum number of non-edges in graphs admitting an induced $F$-decomposition.
\end{abstract}

2010 Mathematics Subject Classification: 05C35, 05C70.

Keywords and Phrases: induced subgraph, edge decomposition, complete multipartite graph, extremal graph theory.

${ }^{*}$ Research supported in part by the Hungarian State and the European Union under the grant TAMOP-4.2.2.A-11/1/KONV-2012-0072 


\section{Introduction}

In this paper we study an extremal problem raised by Bondy and Szwarcfiter [3] in 2013, and extend the results proved in [3, 6, 9]. Throughout, we consider simple undirected graphs, with the central concept of induced decomposition, under which we mean edge-decomposition into induced subgraphs.

\subsection{The problem to be studied}

Definition 1 For two graphs $F$ and $G$, an induced $F$-decomposition of $G$ is a collection $\left\{F_{1}, \ldots, F_{\ell}\right\}$ of induced subgraphs of $G$ such that

- each $F_{i}$ is isomorphic to $F(1 \leq i \leq \ell)$,

- the subgraphs are pairwise edge-disjoint, and

- $\bigcup_{i=1}^{\ell} E\left(F_{i}\right)=E(G)$.

Clearly, for any fixed $F$ different from $K_{2}$, not all graphs $G$ admit an induced $F$-decomposition. Especially, if $F$ is not a complete graph and the induced decomposition exists, then $G$ cannot be complete. Therefore, the following problem was proposed by Bondy and Szwarcfiter.

Problem 2 ([3]) Given a graph $F$ and a positive integer $n \geq|V(F)|$, determine the maximum number $\operatorname{ex}^{*}(n, F)$ of edges in a graph $G$ of order $n$ which admits an induced F-decomposition.

For the asymptotic version of this problem, the following basic result was proved by Cohen and Tuza.

Theorem 3 ([6]) For every graph $F$ we have $\operatorname{ex}^{*}(n, F)=\left(\begin{array}{l}n \\ 2\end{array}\right)-o\left(n^{2}\right)$.

Note that this theorem is analogous to a result of Frankl and Füredi from [8]; the latter one is discussed in a more general framework.

Theorem 3 suggests that in fact the 'complementary' function

$$
\overline{\mathrm{ex}^{*}}(n, F)=\left(\begin{array}{l}
n \\
2
\end{array}\right)-\mathrm{ex}^{*}(n, F)
$$

is the appropriate subject of study, as it is more informative when asymptotic results are desired. 


\subsection{A short summary of earlier asymptotic results}

- If $F$ is a complete graph then, by Wilson's theorem [10, we infer that for each $k$ the equality $\overline{\operatorname{ex}^{*}}\left(n, K_{k}\right)=0$ holds with infinitely many values of $n$. In general, $\overline{\operatorname{ex}^{*}}\left(n, K_{k}\right)=O(n)$ holds for every $k \geq 2$.

- As observed in [3], if $F^{\prime}$ is obtained from $F$ by extending it with an isolated vertex,

$$
\overline{\operatorname{ex}^{*}}(n, F) \leq \overline{\operatorname{ex}^{*}}\left(n, F^{\prime}\right) \leq \overline{\operatorname{ex}^{*}}(n-1, F)+n-1
$$

holds.

- If $F$ is isolate-free and non-complete then $\overline{\operatorname{ex}^{*}}(n, F)=\Omega(n)$. This was proved recently by Halász and Tuza [9]. Particularly, this linear lower bound is asymptotically sharp; that is, $\overline{\operatorname{ex}^{*}}(n, F)=\Theta(n)$ holds if $F$ is a complete bipartite or tripartite graph which is non-complete [9].

- If $F$ is isolate-free, non-complete and not a complete multipartite graph, then $\overline{\operatorname{ex}^{*}}(n, F)=\Omega\left(n^{3 / 2}\right)[9]$. This bound was proved to be asymptotically sharp for $P_{4}, C_{6}, 2 K_{2}$ and $K_{4}-P_{3}$ in [3, 6, 9]. Moreover, as a consequence of results in [1, with Noga Alon we proved that $\overline{\mathrm{ex}^{*}}(n, F)=\Theta\left(n^{3 / 2}\right)$ is valid for every disconnected graph $F$ whose each component is complete bipartite [2].

A more detailed summary of results (not only of the asymptotic ones) and of open problems is given in [4].

Concerning the lower bound $\Omega(n)$ in the third point above, one can observe that the exclusion of isolated vertices is in fact irrelevant.

Proposition 4 If each vertex of $F$ has a non-neighbor (and, in particular, if $F$ has an isolated vertex), then $\overline{\operatorname{ex}^{*}}(n, F) \geq n / 2$.

Proof. Under the given assumption, if $G$ admits an induced $F$-decomposition then every vertex of $G$, too, must have a non-neighbor. 


\subsection{Our main contributions}

The most important contribution of this paper is as follows.

Theorem 5 If $F$ is a complete multipartite graph, then $\overline{\operatorname{ex}^{*}}(n, F)=O(n)$.

Together with the earlier results cited above and with Proposition 4, Theorem 5 immediately implies the following consequences. The first one is the characterization of linear growth, while the second one identifies a gap in the exponents of possible growth functions for $\overline{\operatorname{ex}^{*}}(n, F)$.

Corollary 6 We have $\overline{\operatorname{ex}^{*}}(n, F)=\Theta(n)$ if and only if either $F$ is a complete graph plus at least one isolated vertex, or $F$ is a complete multipartite graph having at least one non-edge, possibly together with some isolated vertices.

Corollary 7 If $1<c<3 / 2$, there exists no $F$ satisfying $\overline{\operatorname{ex}^{*}}(n, F)=\Theta\left(n^{c}\right)$.

In some steps of the constructions we apply design-theoretic methods, in particular 'transversal designs', whose formal definition is given in Section 2. Using those structures the following result will be proved.

Theorem 8 Let $F=K_{a_{1}, \ldots, a_{k}}$, and suppose that there exists a transversal design $T D\left(k, a_{i}\right)$ for each $i=1,2, \ldots, k$. Then the complete multipartite graph $F^{*}=K_{m a_{1}, \ldots, m a_{k}}$ with $m=\prod_{i=1}^{k} a_{i}$ admits an F-decomposition. Moreover, such a decomposition can be encoded with $m^{2}$ integer sequences of length $2 k$ whose $i^{\text {th }}$ and $(k+i)^{\text {th }}$ terms vary between 1 and $a_{i}$.

After some preliminary observations in Section 2, we prove our main result, Theorem 5 in Section 3 . The proof of Theorem 8 is given in Section 4. We close the paper with several open problems and conjectures.

\section{Edge decompositions of complete $k$-partite graphs}

In this section we make some preparations towards the proofs of Theorems 5 and 8 . In the first subsection we recall some important known facts from the theory of transversal designs. In the second subsection we construct decompositions of some restricted classes of graphs - having the same number of partite classes that the fixed given graph $F$ has - which will be applied later for the general results. 


\subsection{Transversal designs}

In general, a transversal design has three parameters: the "order" or "groupsize" $n$, the "blocksize" $k$, and the "index" $\lambda$. For our purpose, however, only the case $\lambda=1$ will be relevant, therefore we disregard the general definition. Hence, the combinatorial structure denoted $\operatorname{TD}(k, n)$ is a triple $(X, \mathcal{G}, \mathcal{B})$, where

1. $X$ is a set of $k n$ elements;

2. $\mathcal{G}=\left(X_{1}, X_{2}, \ldots, X_{k}\right)$ is a partition of $X$ into $k$ classes $X_{i}$ (the groups), each of size $n$;

3. $\mathcal{B}$ is a collection of $k$-element subsets of $X$ (the blocks);

4. every unordered pair of elements from $X$ is contained either in exactly one group or in exactly one block, but not both.

The more widely known structures of Latin squares are arrangements of the numbers $1,2, \ldots, n$ in an $n \times n$ square in such a way that each number occurs precisely once in each row and also in each column. Two Latin squares $\left(a_{i j}\right)_{1 \leq i, j \leq n}$ and $\left(b_{i j}\right)_{1 \leq i, j \leq n}$ are said to be orthogonal if their position-wise concatenation $\left\{a_{i j} b_{i j} \mid 1 \leq i \leq n, 1 \leq j \leq n\right\}$ lists all the $n^{2}$ ordered pairs of $\{1,2, \ldots, n\} \times\{1,2, \ldots, n\}$.

We shall apply the following two facts, the first of which is not very hard to prove.

Lemma 9 (see Theorem 3.18 in [7]) The existence of a TD $(k, n)$ is equivalent to the existence of $k-2$ mutually orthogonal Latin squares of order $n$.

Lemma 10 (Chowla, Erdös and Straus, [5]) The maximum number of mutually orthogonal Latin squares of order $n$ tends to infinity as $n \rightarrow \infty$.

\subsection{Decompositions of complete $k$-partite graphs}

Next, we describe some steps which make it possible to apply transversal designs to the general decomposition problem. We begin with a definition. 
Definition 11 Let $G=(V, E)$ and $F=(X, H)$ be $k$-partite graphs with vertex partitions $V=V_{1} \cup \cdots \cup V_{k}$ and $X=X_{1} \cup \cdots \cup X_{k}$. Assume further that $|V| /|X|=p$ is an integer and that $\left|V_{i}\right|=p\left|X_{i}\right|$ for all $1 \leq i \leq k$. An embedded $F$-decomposition of $G$ is an $F$-decomposition with the further property that each $V_{i}$ is partitioned into $p$ sets,

$$
V_{i}=V_{i, 1} \cup \cdots \cup V_{i, p}
$$

and, for each copy of $F$ in the decomposition, each partite set $X_{i}$ coincides with some $V_{i, j}$ inside $V_{i}$.

Lemma 12 Let $k$ and $a_{1}, \ldots, a_{k}$ be fixed natural numbers. Then, for every sufficiently large integer $p$, the complete multipartite graph $K_{p a_{1}, \ldots, p a_{k}}$ has an embedded $K_{a_{1}, \ldots, a_{k}}$-decomposition.

Proof. Based on Lemma 10 we can choose a threshold value $p_{0}=p_{0}(k)$ such that there exist at least $k-2$ mutually orthogonal Latin squares of order $p$ whenever $p>p_{0}$ holds. Then, by Lemma 9, there exists a transversal design $\operatorname{TD}(k, p)$ of order $p$ and blocksize $k$. Observe that $\operatorname{TD}(k, p)$ is precisely an edge decomposition of the complete $k$-partite graph $K_{p, \ldots, p}$ into copies of the complete graph $K_{k}$. Substituting mutually disjoint sets of cardinality $a_{i}$ for all vertices in the $i^{\text {th }}$ group of $\operatorname{TD}(k, p)$ for $i=1,2, \ldots, k$, each copy of $K_{k}$ becomes a copy of $K_{a_{1}, \ldots, a_{k}}$. Thus, an embedded $K_{a_{1}, \ldots, a_{k}}$-decomposition of $K_{p a_{1}, \ldots, p a_{k}}$ is obtained.

Choosing $p_{0}$ as in the proof above, and letting $a_{i}^{*}=p a_{i}$ for $i=1,2, \ldots, k$ with an arbitrarily fixed $p>p_{0}$, we obtain:

Corollary 13 For every $k$-tuple $\left(a_{1}, \ldots, a_{k}\right)$ of positive integers there exists a k-tuple $\left(a_{1}^{*}, \ldots, a_{k}^{*}\right)$ such that

- $K_{a_{1}^{*}, \ldots, a_{k}^{*}}$ has an embedded $K_{a_{1}, \ldots, a_{k}}$-decomposition;

- a transversal design $T D^{i}=T D\left(k, a_{i}^{*}\right)$ exists for each $i=1,2, \ldots, k$.

The applicability of this approach is demonstrated by Theorem 8 , which was stated in the Introduction and will be proved in Section 4 . 


\section{Proof of the linear upper bound}

In this section we prove Theorem 5 .

Let $F=K_{a_{1}, \ldots, a_{k}}$ be a fixed given graph. With reference to Lemma 12 we choose an integer $p>1$ such that

- $p$ is a multiple of $\prod_{i=1}^{k} a_{i}$,

- $F_{p}:=K_{p a_{1}, \ldots, p a_{k}}$ has an embedded $F$-decomposition.

The general form of Wilson's theorem yields that there exists a modulus $q$ and a residue $r$ such that the complete graph $K_{s q+r}$ has an $F$-decomposition whenever $s \geq s_{0}=s_{0}(F)$ is sufficiently large. Note that, regarding the linear upper bound to be proved, we may assume that $n$ is large. We write $n$ in the form

$$
n=(s q+r) \cdot p+t \quad\left(s \geq s_{0}, \quad 0 \leq t \leq p q-1\right)
$$

and define

$$
n^{\prime}=s q+r
$$

Step 1. Decompose $K_{n^{\prime}}$ into edge-disjoint copies of $F$. Of course, those copies are non-induced subgraphs of $K_{n^{\prime}}$ (unless $F$ itself is a complete graph, which case we disregard).

Step 2. Replace each vertex of $K_{n^{\prime}}$ with an independent set of cardinality $p$. That is, two vertices are adjacent if and only if they belong to different independent sets; in this way the graph

$$
G^{\prime}:=K_{(s q+r) \cdot p}-(s q+r) \cdot K_{p}=K_{n-t}-\frac{n-t}{p} \cdot K_{p}
$$

is obtained. Moreover, each copy of $F$ in $K_{n^{\prime}}$ becomes a copy of $F_{p}$ in $G^{\prime}$. Observe that, although the copies of $F_{p}$ are still non-induced, the $i^{\text {th }}$ vertex class of cardinality $p a_{i}$ is the union of $a_{i}$ independent sets of size $p$ each.

Step 3. In any copy of $F_{p}$, if an independent set $S$ of size $p$ belongs to the $i^{\text {th }}$ vertex class of $F_{p}$, then partition $S$ into $p / a_{i}$ disjoint sets. In this way the $a_{i}$ independent $p$-element sets inside the $i^{\text {th }}$ vertex class together yield a partition of that class into $p$ independent sets of size $a_{i}$ each. 
Step 4. Denoting by $V_{1}, \ldots, V_{k}$ the vertex classes in the copy of $F_{p}$, and by $V_{i, j}$ the sets in the partition obtained $\left(1 \leq i \leq k, 1 \leq j \leq p,\left|V_{i, j}\right|=a_{i}\right.$ for all $i$ and $j$ ), the embedded $F$-decomposition of $F_{p}$ - guaranteed by Lemma 12 and by the choice of $p$ - yields $p^{2}$ copies of $F$ which are induced subgraphs of $G^{\prime}$.

Step 5. Complete the construction with $t$ isolated vertices, to obtain a graph $G$. It is clear that this $G$ admits an induced $F$-decomposition.

It remains to observe that the complement of $G$ has no more than a linear number of edges. There are $\left(\begin{array}{l}t \\ 2\end{array}\right)+t n-t^{2}<t n<p q n$ vertex pairs containing at least one of the $t$ isolated vertices; and the number of non-edges in $G^{\prime}$ is exactly $\frac{n-t}{p} \cdot\left(\begin{array}{l}p \\ 2\end{array}\right)<p n / 2$. Thus,

$$
\overline{\operatorname{ex}^{*}}(n, F) \leq\left(\begin{array}{l}
n \\
2
\end{array}\right)-|E(G)|<(p q+p / 2) \cdot n=O(n) .
$$

This completes the proof of Theorem 5 .

\section{Complete $k$-partite graphs and transversal designs}

Here we prove Theorem 8 . Recall that $F=K_{a_{1}, \ldots, a_{k}}, F^{*}=K_{m a_{1}, \ldots, m a_{k}}$, and $m=\prod_{i=1}^{k} a_{i}$. It is now assumed that there exists a transversal design $\operatorname{TD}\left(k, a_{i}\right)=\left(X^{i}, \mathcal{G}^{i}, \mathcal{B}^{i}\right)$ for every $i=1,2, \ldots, k$, which we shall refer to as $\mathrm{TD}^{i}$ or $\mathrm{TD}^{i}\left(k, a_{i}\right)$. Those $\mathrm{TD}^{i}$ will become important in the last step of the construction.

In order to simplify notation throughout, we write the usual shorthand $\left[a_{i}\right]=\left\{1,2, \ldots, a_{i}\right\}$.

Notation. For $1 \leq i \leq k, 1 \leq p \leq a_{i}$, and $1 \leq q \leq k$, the $p^{\text {th }}$ element in the $q^{\text {th }}$ group $X_{q}^{i}$ of $\operatorname{TD}^{i}\left(k, a_{i}\right)$ will be denoted by $\pi^{i}(p, q)$. (Inside each group the elements are numbered as $1,2, \ldots, a_{i}$.) Note that for all $1 \leq p, p^{\prime} \leq a_{i}$ and all $1 \leq q, q^{\prime} \leq k\left(q \neq q^{\prime}\right)$, a block in $\mathcal{B}^{i}$ containing the $p^{\text {th }}$ element of the $q^{\text {th }}$ group and the $\left(p^{\prime}\right)^{\text {th }}$ element of the $\left(q^{\prime}\right)^{\text {th }}$ group in $\operatorname{TD}^{i}\left(k, a_{i}\right)$ exists and is unique. 
Construction. Let the vertex set of $F^{*}$ be

$$
V=V^{1} \cup \cdots \cup V^{k}, \quad\left|V^{i}\right|=m a_{i} \text { for } i=1, \ldots, k .
$$

Perhaps the best way of thinking about $V^{i}$ is to view it as a $k$-dimensional box composed of unit-cube cells containing $a_{i}$ items each; in the $j^{\text {th }}$ direction the box has size $a_{j}$. In this view we see $a_{i}$ layers orthogonal to the $i^{\text {th }}$ direction, each of those layers consists of $m / a_{i}$ cells, and hence each layer contains precisely $m$ items.

Stating this in a more formal description, we partition each $V^{i}$ into $m$ disjoint sets of cardinality $a_{i}$ (corresponding to the contents of the cells), and represent each of those subsets inside $V^{i}$ with a vector

$$
\mathbf{j}=\left(j_{1}, \ldots, j_{k}\right) \in\left[a_{1}\right] \times \cdots \times\left[a_{k}\right]
$$

of length $k$, so that

$$
V^{i}=\bigcup_{\mathbf{j} \in\left[a_{1}\right] \times \cdots \times\left[a_{k}\right]} V_{\mathbf{j}}^{i} .
$$

In this way a copy of $F$ inside $F^{*}$ is characterized by a $k$-tuple of vectors,

$$
\left(\mathbf{j}^{1}, \ldots, \mathbf{j}^{k}\right) \in\left(\left[a_{1}\right] \times \cdots \times\left[a_{k}\right]\right)^{k},
$$

where

$$
\mathbf{j}^{i}=\left(j_{1}^{i}, \ldots, j_{k}^{i}\right) \in\left[a_{1}\right] \times \cdots \times\left[a_{k}\right]
$$

for $i=1,2, \ldots, k$. Let us refer to this $\left(\mathbf{j}^{1}, \ldots, \mathbf{j}^{k}\right)$ as the detailed representation of the copy in question.

There are $\left(m a_{i}\right)\left(m a_{i^{\prime}}\right)=m^{2} a_{i} a_{i^{\prime}}$ edges of $F^{*}$ between $V^{i}$ and $V^{i^{\prime}}$, and each copy of $F$ contains $a_{i} a_{i^{\prime}}$ edges between those two partite sets. Thus, we have to define $m^{2}$ edge-disjoint copies of $F$ inside $F^{*}$. The key point is how to shuffle the vertex sets of the $m^{2}$ copies to ensure that those subgraphs compose a partition of the edge set of $F^{*}$.

The copies of $F$ will be encoded with vectors of length $2 k$ (i.e., a suitable collection of $k^{2}$-dimensional vectors will be encoded with $(2 k)$-dimensional ones), which we shall view as concatenations of two vectors

$$
(\mathbf{b}, \mathbf{c}) \in\left(\left[a_{1}\right] \times \cdots \times\left[a_{k}\right]\right)^{2}
$$

where

$$
\mathbf{b}=\left(b_{1}, \ldots, b_{k}\right) \in\left[a_{1}\right] \times \cdots \times\left[a_{k}\right],
$$




$$
\mathbf{c}=\left(c_{1}, \ldots, c_{k}\right) \in\left[a_{1}\right] \times \cdots \times\left[a_{k}\right]
$$

are vectors of length $k$.

We identify a copy of $F$ for any given $(\mathbf{b}, \mathbf{c})$ in the way detailed next.

- Generally speaking, the first $k$ coordinates (i.e., those of $\mathbf{b}$ ) specify a $k$-tuple of layers (one layer in each of the boxes $V^{i}$ ) that means a copy of $K_{m, \ldots, m}$; and the role of $\mathbf{c}$ is similar, but "rotating" by 1 among the boxes, e.g. while the first coordinate of $\mathbf{b}$ will provide a data for $V^{1}$, the first coordinate of $\mathbf{c}$ will be relevant for $V^{2}$, hence specifying a second copy of $K_{m, \ldots, m}$. After that, the transversal designs will be used for defining further $k-2$ layers inside each $V^{i}$. Having all this at hand, exactly $k$ layers are determined inside each box, and all the intersections of those layers in the different boxes together uniquely identify a copy of $F$ associated with $(\mathbf{b}, \mathbf{c})$.

- The $i^{\text {th }}$ coordinate $b_{i}$ of $\mathbf{b}$ determines that the $i^{\text {th }}$ partite set of $F$ (inside $\left.V^{i}\right)$ must be one of those $V_{\mathbf{j}}^{i}$ which have $j_{i}=b_{i}$. This equivalently means that $j_{i}^{i}=b_{i}$ holds in the detailed representation. (Recall that $\mathbf{j}^{i}$ has the form $\mathbf{j}^{i}=\left(j_{1}^{i}, \ldots, j_{k}^{i}\right)$.)

- The $i^{\text {th }}$ coordinate $c_{i}$ of $\mathbf{c}$ determines the $i^{\text {th }}$ coordinate $j_{i}^{i+1}$ of the next $\mathbf{j}^{i+1}$ in the detailed representation. (For $i=k$ we mean $\mathbf{j}^{k+1}=\mathbf{j}^{1}$.)

- By the definition of the transversal designs $\operatorname{TD}^{i}\left(k, a_{i}\right)$, there is a unique block $B^{i}=B^{i}(\mathbf{b}, \mathbf{c}) \in \mathcal{B}^{i}$ such that both $\pi^{i}\left(b_{i}, i\right) \in B^{i}$ and $\pi^{i}\left(c_{i}, i+1\right) \in$ $B^{i}$. (Also here, the successor $i+1$ of $i=k$ is meant to be 1.) Then the $i^{\text {th }}$ coordinate of the copy of $F$ associated with $(\mathbf{b}, \mathbf{c})$ in $V^{i^{\prime}}$, that is $j_{i}^{i^{\prime}}$ in the representation $\left(\mathbf{j}^{1}, \ldots, \mathbf{j}^{k}\right)$, is defined as $j_{i}^{i^{\prime}}=B^{i} \cap X_{i^{\prime}}^{i}$ for all $i^{\prime}$. This is our defining rule for $i^{\prime} \notin\{i, i+1\}$, but the formula is valid also for $i^{\prime} \in\{i, i+1\}$ because this was the way we selected $B^{i}$.

Verification of requirements. It is clear that precisely $m^{2}$ copies are defined in this way. Since the total number of edges in those copies is equal to that in $F^{*}$, the proof will be done if we show that each edge of $F^{*}$ is contained in some copy of $F$. This is equivalent to saying that each edge $v_{i} v_{i^{\prime}}\left(v_{i} \in V^{i}, v_{i^{\prime}} \in V^{i^{\prime}}\right)$ determines all the $k^{2}$ coordinates of some $k$-tuple of vectors $\left(\mathbf{j}^{1}, \ldots, \mathbf{j}^{k}\right)$. The property is valid indeed, as can be seen from the following considerations. 
The positions of $v_{i}$ and $v_{i^{\prime}}$ determine the representing vectors $\mathbf{j}^{i}$ and $\mathbf{j}^{i^{\prime}}$ of the $a_{i^{-}}$-element and $a_{i^{\prime}}$-element sets (cells) containing $v_{i}$ and $v_{i^{\prime}}$, respectively. This determines $k$ pairs of coordinates:

$$
\left(j_{1}^{i}, j_{1}^{i^{\prime}}\right), \quad\left(j_{2}^{i}, j_{2}^{i^{\prime}}\right), \quad \ldots, \quad\left(j_{k}^{i}, j_{k}^{i^{\prime}}\right) .
$$

The pair $\left(j_{\ell}^{i}, j_{\ell}^{i^{\prime}}\right)$ uniquely determines the block $B^{\ell}=B^{\ell}(\mathbf{b}, \mathbf{c}) \in \mathcal{B}^{\ell}$ in $\mathrm{TD}^{\ell}\left(k, a_{\ell}\right)$ for $\ell=1, \ldots, k$; this $B^{\ell}$ is the unique block containing the $\left(j_{\ell}^{i}\right)^{\text {th }}$ element of the $i^{\text {th }}$ block and the $\left(j_{\ell}^{i^{\prime}}\right)^{\text {th }}$ element of the $\left(i^{\prime}\right)^{\text {th }}$ block in $\operatorname{TD}^{\ell}\left(k, a_{\ell}\right)$. Thus, all the $k^{2}$ coordinates of the vectors $\mathbf{j}^{1}, \ldots, \mathbf{j}^{k}$ can be computed from those $k$ blocks by the rule $j_{\ell}^{i}=B^{\ell} \cap X_{i}^{\ell}$.

This completes the proof of the theorem.

\section{Concluding remarks}

In this paper we studied the growth of the number of edges in graphs which admit edge decompositions into induced subgraphs isomorphic to a given complete multipartite graph. We proved a linear upper bound on the number of non-edges. More explicitly, our construction yields the following.

Theorem 14 If $n$ satisfies the following three conditions:

- $\left(\begin{array}{l}n \\ 2\end{array}\right)$ is divisible by the number $\sum_{1 \leq i<i^{\prime} \leq k} a_{i} a_{i^{\prime}}$ of edges in $F$,

- $n-1$ is divisible by the greatest common divisor of the vertex degrees $\left(\sum_{i=1}^{k} a_{i}\right)-a_{1},\left(\sum_{i=1}^{k} a_{i}\right)-a_{2}, \ldots,\left(\sum_{i=1}^{k} a_{i}\right)-a_{k}$,

- $n>n_{0}$ for some threshold value $n_{0}=n_{0}\left(a_{1}, \ldots, a_{k}\right)$,

then the complete $n$-partite graph $K_{m, \ldots, m}$ with $m=\prod_{i=1}^{k} a_{i}$ admits an edge decomposition into induced subgraphs isomorphic to $F$.

Comparison with the results from [9] identifies a gap in the exponent of $n$; this can be expressed in the following way, where the first case is trivial and the second case is taken from Wilson's theorem. 
Let $F$ be a graph without isolated vertices.

- If $F=K_{2}$, then $\overline{\mathrm{ex}^{*}}(n, F)=0$.

- If $F$ is a complete graph of order at least 3, then $\overline{\operatorname{ex}^{*}}(n, F)$ oscillates between 0 and $\Theta(n)$, reaching both extremities on sets of positive upper density.

- If $F$ is a complete multipartite graph, but not a complete graph, then $\overline{\mathrm{ex}^{*}}(n, F)=\Theta(n)$.

- If $F$ is not a complete multipartite graph, then $\overline{\operatorname{ex}^{*}}(n, F)=\Omega\left(n^{3 / 2}\right)$.

The next task could be to identify further gaps in the exponent of $n$ describing the growth of $\overline{\operatorname{ex}^{*}}(n, F)$, if there are any. We believe that not all reals (and not all rational numbers) between $3 / 2$ and 2 can occur as exponents for some $F$, and also that the gap between $n$ and $n^{3 / 2}$ is not the only one. This is expressed in the following sequence of conjectures.

Conjecture 15 There exist graphs $F$ for which

$$
\lim _{n \rightarrow \infty} \frac{\overline{\operatorname{ex}^{*}}(n, F)}{n^{3 / 2}}=\infty \text {. }
$$

Conjecture 16 For every non-complete graph $F$, without isolated vertices, there exists a constant $c=c(F)$ such that $\overline{\operatorname{ex}^{*}}(n, F)=\Theta\left(n^{c}\right)$.

Conjecture 17 The constant $c=c(F)$ in Conjecture 16 is rational for every graph $F$.

Conjecture 18 Every non-complete graph $F$ without isolated vertices satisfies the asymptotic equality $\overline{\mathrm{ex}^{*}}(n, F)=\Theta\left(n^{2-1 / t}\right)$ where $t=t(F)$ is a positive integer.

If Conjecture 18 is true, the next question is: Which structural property of $F$ determines $t(F)$ ?

Conjecture 19 Every non-complete graph $F$ without isolated vertices, the quotient

$$
\frac{\overline{\operatorname{ex}^{*}}(n, F)}{n^{2-1 / t}}
$$

tends to a positive limit as $n$ gets large, for some positive integer $t=t(F)$. 
If this is true, we would be interested in the exact value of the limit.

Problem 20 Verify Conjectures 18 and 19 for all graphs on at most six vertices.

The smallest open case for Conjecture 18 is $F=C_{5}$, while the one for Conjecture 19 is $F=2 K_{2}$.

Hypergraphs. It is very natural to extend the above questions to $r$-uniform hypergraphs $(r \geq 3)$, but rather too little is known so far about the analogous function $\overline{\mathrm{ex}^{*}}(n, \mathcal{F})$. Already the study of some very small examples may provide interesting pieces of information.

\section{References}

[1] N. Alon, A. Moitra and B. Sudakov, Large induced matchings and their applications, Journal of the European Mathematical Society (JEMS) 15 (2013), 1575-1596.

[2] N. Alon and Zs. Tuza, unpublished, July 2014.

[3] J. A. Bondy and J. L. Szwarcfiter, Induced decompositions of graphs, Journal of Graph Theory 72 (2013), 462-477.

[4] Cs. Bujtás and Zs. Tuza, Problems and results on induced subgraph decompositions of graphs, Proc. ASCONIKK 2014: Extended Abstracts III. Future Internet Technologies, ISBN 978-963-396-048-6, University of Pannonia, Veszprém, 2014, pp. 11-18.

[5] S. A. Chowla, P. Erdős and E. G. Straus, On the maximal number of pairwise orthogonal latin squares of a given order, Canad. J. Math. 13 (1960) 204-208.

[6] N. Cohen and Zs. Tuza, Induced decompositions of highly dense graphs, Journal of Graph Theory (2014), DOI: 10.1002/jgt.21792, in print.

[7] C. J. Colbourn and J. H. Dinitz (eds.), Handbook of Combinatorial Designs, 2nd edition, Chapman \& Hall/CRC, 2007. 
[8] P. Frankl and Z. Füredi, Colored packing of sets, Annals of Discrete Math. 34 (1987), 165-178.

[9] V. Halász and Zs. Tuza, Asymptotically optimal induced decompositions, Applicable Analysis and Discrete Mathematics 8 (2014), 320-329.

[10] R. M. Wilson, Decompositions of complete graphs into subgraphs isomorphic to a given graph, Proc. $5^{\text {th }}$ British Combinatorial Conference, Congressus Numerantium XV (1976), 647-659. 